\title{
The Effect of a Short Planning Horizon on Intertemporal Consumption Choices
}

\author{
EnRICA CARBOnE*ANd Gerardo Infante ${ }^{\dagger}$
}

March 29, 2014

\begin{abstract}
Experiments on intertemporal consumption typically show that people have difficulties in optimally solving this kind of problems. Previous studies have focused on contexts in which agents are faced with risk on future income and have to plan over long horizons. We present an experiment comparing decision making under certainty, risk and ambiguity, over a shorter life-cycle. Results show that planning in the ambiguity treatment is markedly different than in the risk condition and it is characterized by a significant pattern of under-consumption.

Keywords Intertemporal Consumer Choice - Life Cycle · Risk • Ambiguity $\cdot$ Laboratory Experiments

JEL Classification D12 - D91 - D81 - C91 - C92

*Seconda Università di Napoli, Corso Gran Priorato di Malta n.1, 81043 Capua (CE), ITALY, tel.+39-339-5491936 fax. +39-0823-622984; email: enrica.carbone@gmail.com

†Università degli Studi di Bari
\end{abstract}




\section{Introduction}

The question of how people cope with solving dynamic optimization problems has been often tested in economics. Several contributions in the literature, including experimental and empirical studies, have shown how people may have difficulties in optimally solving intertemporal consumption/saving problems ${ }^{1}$. Results have generally shown that participants fail to optimise lifetime utility, in some cases deviating significantly from the optimal consumption strategy (Hey and Dardanoni (1988); Ballinger et al. (2003, 2011); Carbone and Hey (2004) as well as Brown et al. (2009)). Other contributions have found evidence of how learning and cognitive abilities may play an important role in improving intertemporal planning ${ }^{2}$.

Experiments on intertemporal consumption/saving problems have typically involved making decisions under risk on the distribution of income (over the life-cycle). In all these cases, participants have knowledge of the stochastic process determining income, no matter the specific procedure (or mechanism) employed. In other words, participants know in advance, or are able to easily determine the probability of receiving a specific level of income (or income shock). Alternatively, such stochastic process could be completely unknown to participants, who would then have to plan under

\footnotetext{
${ }^{1}$ For a review see, among others, Hall (1978), Deaton (1992), Browning and Lusardi (1996). This studies also contribute in underlining problems of misspecification and the necessity for better specializations of the general model, e.g. taking better account of precautionary motives (see also Carroll (1997)) or time non separable preferences (See, among others, Dynan (2000); Guariglia and Rossi (2002); Carrasco et al. (2005))

${ }^{2}$ For an account see Fehr and Zych (1998); Ballinger et al. (2003); Carbone and Hey (2004); Hey (2008); Brown et al. (2009); Ballinger et al. (2011)
} 
ambiguity. This would entail having some prior beliefs about probabilities and a mechanism to update them. It would not be unreasonable to think that many people everyday are faced with consumption/saving decisions under ambiguity about future income. As far as we know, there are no studies that test experimentally people's ability to solve this kind of problems in this specific context.

Other studies also report on people's difficulties in planning ahead, possibly due to the length of the planning horizon (see Carbone and Hey (2004); Hey and Panaccione (2011) or Hey and Knoll (2007, 2011)). In particular, Ballinger et al. (2003) and Carbone and Hey (2004) include a discussion on the estimation of the planning horizon that participants seem to actually use to solve the inter-temporal consumption problem. They find evidence of myopic behaviour in both cases of life-cycles of 25 (Carbone and Hey, 2004) and 60 periods (Ballinger et al., 2003). These authors conclude that not only people may be short-sighted relative to the optimal planning horizon, but that there seems to be a significant variability across subjects. For this reason we decided to run this test with a relatively short horizon. Intuitively, shorter planning horizons might allow agents to reach the optimal solution more easily.

The aim of this experiment is to explore how subjects solve a stochastic optimisation problem in three different decision-making contexts: certainty, risk and ambiguity, in the specific case of a very short life-cycle. Results show that planning in the ambiguity treatment is markedly different than 
in the risk condition. We also find that even in presence of an (unusually) short planning horizon, participants still seem to have difficulties in finding the optimal solution.

The theoretical background for this study is described in Section 2. Section 3 presents the experimental design while results are analyzed in Section 4 and discussed in Section 5.

\section{Theory}

Consider an agent living for a discrete number of periods $(T)$ and having intertemporal preferences represented by the Discounted Utility model with a discount rate equal to zero. In each period, she receives utility from consumption; utility is assumed to have a functional form of the CARA type:

$$
U(c)=\left(k-\frac{e^{-\rho c}}{\rho}\right) \alpha,
$$

where " $\mathrm{c} "$ is consumption, $\alpha$ and $k$ are scaling factors.

In the case of decision making under risk, the objective of our agent is then to maximize the expected lifetime utility, that is ${ }^{3}$

$$
\max E_{t}\left[\sum_{t=1}^{T} \beta U\left(c_{t}\right)\right]
$$

subject to

\footnotetext{
${ }^{3}$ Having set the discount rate equal to zero, $\beta$ equals 1 , so the same can be expressed by: $E\left(U\left(c_{t}\right)+U\left(c_{t+1}\right)+\cdots+U(T)\right)$.
} 


$$
w_{t+1}=a_{t+1}+y=(1+r)\left(w_{t}-c_{t}\right)+y
$$

where $w$ is available wealth, $a$ represents available assets or savings at the beginning of period $t+1$ and $y$ is income. In each period of her lifecycle, the agent receives either a high or a low income, with probabilities $p=q=0.5$. The rate of return is known and held fixed during the lifecycle. Also, borrowing is not allowed, that is, wealth must always be greater or at most equal to zero. Finally, the agent has no bequest motives, that is, any savings are lost after the last period $(T)$. The problem is then to choose the sequence of consumption (from period 1 to period $T$ ) that maximizes (1).

The optimal strategy under risk assumes Expected Utility (EU) decisionmakers who work with the true objective probabilities. Under ambiguity, as we have implemented it, subjects do not know the true probabilities and therefore EU cannot be applied. There are many models of behavior under ambiguity and we choose the simplest - that is, Subjective Expected Utility (SEU) theory, assuming that subjective probabilities are 0.5.

The standard procedure to solve this kind of problems is to use Dynamic Programming ${ }^{4}$. The Bellman Equation of the problem has been determined as

$$
V_{t}\left(w_{t}\right)=U\left(c_{t}^{*}\right)+E\left[V_{t+1}\left(w_{t+1}^{*}\right)\right]
$$

\footnotetext{
${ }^{4}$ See among others Deaton (1992) and Stokey et al. (1989)
} 
where $V_{t}$ is the value function, $w_{t}$ represents available wealth and $E$ is the expectation operator ${ }^{5}$. The value function establishes a recursive relation between current and future decisions. The expectation is resolved by considering the two possible events: low income and high income. Wealth in period $t+1$ is optimal because it is determined by the (optimal) consumption choice in $t$. Using backward induction, the agent starts from the last period $(T)$, where the optimal solution is obviously to consume all wealth, then moves backwards period by period, choosing the optimal level of consumption which maximizes the value function of that period, until the first period is reached. This allows the determination of optimal consumption as a function of wealth $\left(w_{t}\right)$ and time $(t)$.

Some restrictions have been imposed on variables. In particular, as anticipated, borrowing is not allowed $\left(w_{t} \geq 0\right)$ and all variables are rounded to the second decimal figure. For this reason, while in the case of certainty it was possible to determine the exact solution of the problem, in the case of risk and ambiguity a numerical solution (also using interpolation) had to be used $^{6}$.

\section{Experimental Design}

The experiment is composed of three treatments, denominated "certainty", "risk" and "ambiguity". Participants were randomly assigned to each treatment. The rate of interest $(r)$ was fixed at 0.4 , while income was set equal to

\footnotetext{
${ }^{5}$ Starred variables indicate optimal choices

${ }^{6}$ The optimization programs were written using Maple
} 
10 tokens in the certainty case and to 5 and 15 tokens in the cases of risk and ambiguity. The probability of high or low income was equal to 0.5 . The parameters of the utility function, presented in the experiment as a "conversion function" from tokens to money, were set as follows: $\rho=0.1 ; \alpha=0.45$ and $k=10$. The experiment was run at LABSI at the University of Siena. Thirty undergraduate students took part in three sessions, one for each treatment. The experiment was programmed and conducted with the software z-Tree (Fischbacher, 2007). Each session involved playing five sequences, each one composed of five periods. In each period of a sequence subjects were asked to decide how much to convert out of their available tokens (the sum of income, previous savings and interest) knowing that any tokens not converted would yield interest. In the risk and ambiguity treatments, in each period income was determined by a random draw from an opaque bag containing equal numbers of two coloured balls. In the case of risk, at the beginning of the experiment, one participant was asked to publicly open the bag and count the balls ${ }^{7}$. After each draw, the ball was placed back into the bag so as to not alter the probability of future draws. Instructions also clarified that any savings left at the end of the last period would be worthless. In order to allow participants to check the consequences of their decisions, a calculator was made available in each period ${ }^{8}$. At the beginning of each period and at the end of a sequence, participants were shown a table summarizing the consequences of their decisions reporting income, available wealth, conversion, savings, interest gained and earnings (in the previous period or in the

\footnotetext{
${ }^{7}$ This was omitted in the case of ambiguity

${ }^{8}$ Participants were also provided with tables showing some examples of conversions and of the interest mechanism.
} 
whole sequence, period by period). Participants could enter numbers with up to two decimal digits for conversion. At the end of the experiment one of the five sequences played was randomly selected for payment using a public procedure.

\section{Results and discussion}

Table 1 presents a summary of the experiment showing, for each treatment, three different types of information. In the top part of the table there is a comparison between the theoretical maximum utility (labeled "Opt. Ut.") and the average total utility achieved by participants in that treatment (along with its standard deviation $)^{9}$. Results show that all deviations are negative. More interestingly, the second part of Table 1 shows that they are also all statistically significant, according to one-sample parametric and non-parametric tests (t-test and signed rank test), which suggests that participants, on average, did not maximize utility. Also, deviations in the case of decision making under ambiguity are generally slightly greater than those in other treatments. Moreover, deviations show a similar pattern of variability across treatments: usually higher in the first and second sequences and lower in the following repetitions. Finally, the third part of Table 1 shows the root mean squared deviation (RMSD) for each sequence and each treatment, in the cases of unconditional and conditional optimum. The main difference here is the point of reference: while unconditional optimum represents the solution to the intertemporal problem and is calculated on optimal wealth (hence assuming

\footnotetext{
${ }^{9}$ Here we refer to the "ex-post" optimum, i.e. the optimal solution calculated after income realizations
} 
optimal behaviour throughout the lifecycle), conditional optimum is computed based on actual wealth, and is traditionally assumed to incorporate a measure of improvement of behaviour ${ }^{10}$. As Table 1 shows, this index tends to decrease across sequences, suggesting an improvement in strategy during the experiment.

In general the presence of significant deviations from unconditional optimum suggests that participants did not use the optimal strategy. Moreover this analysis shows that subjects in the ambiguity treatment deviate more from optimum.

\subsection{Estimated planning horizon}

Following Ballinger et al. (2003) and Carbone and Hey (2004) the apparent planning horizon used by participants has been estimated, sequence by sequence for each treatment. Planning horizons have been estimated in two stages. First, the optimal path for each possible planning horizon has been calculated using the optimal consumption functions. The apparent planning horizon was then identified as the one minimizing the mean squared deviation from optimal consumption (both unconditional and conditional optima were considered). The Null Hypothesis that the average actual and optimal planning horizons are the same has then been tested using both the Signed Rank test and the t-test ${ }^{11}$ (one-sample tests). Results show that in the cases of cer-

\footnotetext{
${ }^{10}$ Further discussions on the concepts of conditional and unconditional optima can be found in Ballinger et al. (2003); Carbone and Hey (2004).

${ }^{11}$ Tables ??, ?? and ??, relative to the three treatments are reported in Appendix ??. The results of the statistical tests are available on request
} 
tainty and risk the average apparent horizon is always significantly shorter than optimal. In the ambiguity case, the average actual planning horizon seems to be longer than the one used in other treatments. Indeed, statistical tests confirm that there is no statistical difference with the optimal horizon in the first three sequences, when considering unconditional optimum and in two cases when considering the conditional optimum.

This result is somewhat at odds with initial findings, suggesting that in the case of decision making under ambiguity participants deviated more from maximum utility. A possible explanation for this could be that given the very short length of the lifecycle, some (possibly extreme) strategies might cause biased estimations. An "informal" analysis of this hypothesis has highlighted that saving "aggressively" (i.e. most or all of available wealth) in the first/second periods seems to result in an estimated planning horizon of four/five periods. This phenomenon seems to be more evident when looking at data from the risk and ambiguity treatments. For this reason and in order to investigate the existence of regularities that influenced the estimation of actual planning horizons, the distribution of the fraction of consumption over available wealth, for all treatments has been analyzed.

\subsection{Consumption-to-wealth ratios}

For each treatment and each sequence, the comparison between the optimal consumption-to-wealth ratio $\left(c^{*} / w^{*}\right)$ and the average of actual ratios has 
been computed ${ }^{12}$. Graphs in Figure 1 compare treatments with respect to deviations from optimal ratios. The "x-axis" represents a deviation equal to zero, while positive and negative values can be interpreted as instances of over- and under-consumption. An interesting finding, immediately visible from the graphs, is that in the case of ambiguity, ratios are consistently below zero (implying an average under-spending with respect to optimum) and consistently below the other two treatments. This finding, together with the pattern described at the end of the previous subsection, might help explain the apparent contradiction between deviations from optimum and estimated planning horizons.

\subsection{Comparing risk and ambiguity}

Table 2 and table 3 show the comparison between risk and ambiguity in each period of each sequence and when pooling data together, respectively. Two-sample tests (Wilcoxon-Mann-Whitney and t-test) have been carried out on the average deviations from conditional optimum. Results reported in table 2 show that, in periods 1 to $4^{13}$, the behaviour under ambiguity is different from the behaviour under risk. More specifically, in the ambiguity treatment we observe a trend of under-consumption, with a bigger average deviation from conditional optimum (in absolute value) compared to the risk case. In the risk treatment behaviour seems to be more "unstable": in some cases actual consumption is above conditional optimum, in others it falls

\footnotetext{
${ }^{12} \mathrm{~A}$ table reporting optimal and average actual ratios (with their standard deviations) and deviations, is available on request.

${ }^{13}$ Period 5 is not relevant because participants were clearly instructed that "leftovers" after the last periods would be lost
} 
below it. Table 2 shows that risk and ambiguity treatments are significantly different in sequence 2 (periods 3 and 4 ) and sequence 3 (periods 2 and 3). However, when looking at the general picture, average deviations in cases of risk and ambiguity are generally very far apart (different). Table 3, where data from all sequences are pooled together, shows that aside from period one, the ambiguity treatment is always significantly different from the risk treatment. While the behaviour under risk exhibits over-consumption at the beginning of the horizon (and under-consumption in the second half, as typically reported in the literature), the ambiguity treatment exhibits a persistent pattern of (average) under-consumption. Also, in the ambiguity case, average deviations are almost always negative in sequences 1 to 3 , while in sequences 4 and 5 they become positive in the first period and negative elsewhere. This "learning" effect in the last two sequences, can be interpreted in the following way: in Subjective Expected Utility (SEU) theory, subjects have subjective probabilities which may or may not be the true probabilities. As they get more information about the possible states of the world ${ }^{14}$, they update their beliefs. By the time they got to the last round of the last sequence, they had observed 25 draws from the bag. By then, they would have a pretty good idea of the true probabilities. Obviously, we cannot get inside the subjects minds and observe their subjective probabilities at each stage, but we can make two guesses at what they might be thinking. One guess is that, in the absence of information to the contrary, they would start by assuming that each state was equally likely; evidence from the draws

\footnotetext{
${ }^{14}$ Which was the case in this experiment as there was a fresh draw from the ambiguous bag after each round of each sequence
} 
would conform that. The second guess is that the probability of the good state was low, and this would steadily be revised upwards in the light of the observations, this seems to be the case of our experimental subjects. We should note that if we use SEU to explain the behavior of our subjects, then it is all to do with their subjective probabilities; there is no parameter of ambiguity aversion.

\section{Conclusions}

We have presented an experiment designed to study intertemporal consumption choices, in the cases of certainty, risk, and ambiguity about future income, over a short planning horizon. Results show that even when faced with an unusual short life-cycle participants failed to plan optimally, in all treatments.

More interestingly, results show that subjects behaviour in the ambiguity treatment was markedly different from that in the case of risk. Not having information about the distribution of future income seems to have triggered significant savings across most of the sequences. In particular, in the first three sequences of the experiment participants have (on average ) underconsumed (with respect to conditional optimum) throughout the lifecycle. This result is strikingly different from what has been typically reported in the literature. However, by the last two sequences the pattern of deviations has shifted to average over-consumption, in the early periods of the lifecycle, followed by under-consumption in later ones. We believe that this result is 
directly connected to the learning process about the (unknown) probabilities which seem to have induced more precautionary savings out of wealth available for consumption. 


\section{References}

Ballinger, T., Palumbo, M., and Wilcox, N. (2003). Precautionary saving and social learning across generations: an experiment. The Economic Journal, 113(490):920-947.

Ballinger, T. P., Hudson, E., Karkoviata, L., and Wilcox, N. T. (2011). Saving behavior and cognitive abilities. Experimental Economics, 14(3):349374.

Brown, A., Chua, Z., and Camerer, C. (2009). Learning and visceral temptation in dynamic saving experiments. Quarterly Journal of Economics, 124(1):197-231.

Browning, M. and Lusardi, A. (1996). Household saving: Micro theories and micro facts. Journal of Economic Literature, 34(4):1797-1855.

Carbone, E. and Hey, J. (2004). The effect of unemployment on consumption: an experimental analysis. The Economic Journal, 114(497):660-683.

Carrasco, R., Labeaga, J. M., and David Lpez-Salido, J. (2005). Consumption and habits: Evidence from panel data. The Economic Journal, 115(500):144-165.

Carroll, C. D. (1997). Buffer-stock saving and the life cycle/permanent income hypothesis. Quarterly journal of Economics, 112(1):1-56.

Deaton, A. (1992). Understanding Consumption (Clarendon Lectures in Economics). Oxford University Press.

Dynan, K. E. (2000). Habit formation in consumer preferences: Evidence from panel data. The American Economic Review, 90(3):391-406.

Fehr, E. and Zych, P. K. (1998). Do addicts behave rationally? Scandinavian Journal of Economics, 100(3):643-662.

Fischbacher, U. (2007). z-tree: Zurich toolbox for ready-made economic experiments. Experimental Economics, 10(2):171-178.

Guariglia, A. and Rossi, M. (2002). Consumption, habit formation, and precautionary saving: evidence from the british household panel survey. Oxford Economic Papers, 54:1-19.

Hall, R. (1978). Stochastic implications of the life cycle-permanent income hypothesis: Theory and evidence. The Journal of Political Economy, 86(6):971-987. 
Hey, J. (2008). Exploring the experimental economics approach in pensions. Working Paper 43, Department for Work and Pensions.

Hey, J. and Dardanoni, V. (1988). Optimal consumption under uncertainty: An experimental investigation. The Economic Journal, 98(390):105-116.

Hey, J. and Knoll, J. A. (2007). How far ahead do people plan? Economic Letters, 96(1):8-13.

Hey, J. and Knoll, J. A. (2011). Strategies in dynamic decision making an experimental investigation of the rationality of decision behaviour. Journal of Economic Psychology, 32(3):399-409.

Hey, J. and Panaccione, L. (2011). Dynamic decision making: what do people do? Journal of Risk and Uncertainty, 42(2):85-123.

Stokey, N. L., Lucas, R. E., and Prescott, E. (1989). Recursive Methods in Economic Dynamics. Harvard University Press. 


\section{Tables}

Table 1: Summary of Treatments

\begin{tabular}{|c|c|c|c|c|c|}
\hline & \multicolumn{5}{|c|}{ Certainty } \\
\hline & Seq 1 & Seq 2 & Seq 3 & Seq 4 & Seq 5 \\
\hline Opt. UT & 15.0512 & 15.0512 & 15.0512 & 15.0512 & 15.0512 \\
\hline AVG UT & 13.0315 & 13.4179 & 14.0075 & 14.6501 & 14.3830 \\
\hline s.d. & 2.8566 & 1.9157 & 1.5755 & 0.2788 & 0.7570 \\
\hline \multirow{2}{*}{ Deviation } & -2.0197 & -1.6333 & -1.0437 & -0.4011 & -0.6682 \\
\hline & \multicolumn{5}{|c|}{ H0: AVG UT=Opt.UT } \\
\hline t-test $(t=)$ & -2.2359 & -2.6962 & -2.0949 & -4.5500 & -2.7913 \\
\hline \multirow[t]{2}{*}{ signed $\operatorname{rank}(\mathrm{z}=)$} & -2.8030 & -2.8030 & -2.8030 & -2.8030 & -2.8030 \\
\hline & \multicolumn{5}{|c|}{ RMSD } \\
\hline unc.opt. & 10.62 & 10.66 & 9.22 & 3.85 & 4.64 \\
\hline \multirow[t]{3}{*}{ cond.opt. } & 9.11 & 6.45 & 5.19 & 2.32 & 2.97 \\
\hline & \multicolumn{5}{|c|}{ Risk } \\
\hline & Seq 1 & Seq 2 & Seq 3 & Seq 4 & Seq 5 \\
\hline Opt. UT & 16.2700 & 16.0500 & 15.5800 & 15.2300 & 14.4200 \\
\hline AVG UT & 13.0700 & 14.7528 & 15.0037 & 14.5218 & 13.1952 \\
\hline s.d. & 3.0791 & 0.8527 & 0.8205 & 0.7353 & 0.7713 \\
\hline \multirow[t]{2}{*}{ Deviation } & -3.2000 & -1.2972 & -0.5763 & -0.7082 & -1.2248 \\
\hline & \multicolumn{5}{|c|}{ H0: AVG UT=Opt.UT } \\
\hline t-test $(\mathrm{t}=)$ & -3.2829 & -4.8105 & -2.2213 & -3.0457 & -5.0212 \\
\hline \multirow[t]{2}{*}{ signed rank $(\mathrm{z}=)$} & -2.8050 & -2.8030 & -1.7840 & -2.8050 & -2.8050 \\
\hline & \multicolumn{5}{|c|}{ RMSD } \\
\hline unc.opt. & 18.01 & 7.29 & 6.87 & 6.58 & 5.51 \\
\hline \multirow[t]{3}{*}{ cond.opt. } & 10.14 & 4.20 & 3.95 & 3.76 & 3.45 \\
\hline & \multicolumn{5}{|c|}{ Ambiguity } \\
\hline & Seq 1 & Seq 2 & Seq 3 & Seq 4 & Seq 5 \\
\hline Opt. UT & 16.0501 & 16.0974 & 12.2909 & 13.9571 & 15.2293 \\
\hline AVG UT & 12.6472 & 12.9356 & 10.2075 & 12.8590 & 13.8737 \\
\hline s.d. & 3.1820 & 1.6900 & 0.8572 & 0.9386 & 0.8564 \\
\hline \multirow[t]{2}{*}{ Deviation } & -3.4029 & -3.1618 & -2.0834 & -1.0981 & -1.3555 \\
\hline & \multicolumn{5}{|c|}{ H0: AVG UT=Opt.UT } \\
\hline $\mathrm{t}$-test $(\mathrm{t}=)$ & -3.3819 & -5.9162 & -7.6862 & -3.6998 & -5.0053 \\
\hline \multirow[t]{2}{*}{ signed $\operatorname{rank}(\mathrm{z}=)$} & -2.8030 & -2.8030 & -2.8030 & -2.4970 & -2.8030 \\
\hline & \multicolumn{5}{|c|}{ RMSD } \\
\hline unc.opt. & 20.41 & 8.97 & 5.00 & 7.64 & 6.30 \\
\hline cond.opt. & 13.36 & 6.98 & 3.90 & 4.59 & 3.70 \\
\hline
\end{tabular}


Table 2: Comparing Risk and Ambiguity Sequence by Sequence

\begin{tabular}{|c|c|c|c|c|c|c|}
\hline \multirow[b]{2}{*}{ Sequence } & & \multicolumn{5}{|c|}{ Periods } \\
\hline & & 1 & 2 & 3 & 4 & 5 \\
\hline \multirow{4}{*}{1} & AVG (risk) & 0.58 & -0.44 & -3.892 & -9.011 & -0.001 \\
\hline & AVG (amb) & -2.942 & -4.844 & -8.90527 & -11.51 & -0.004 \\
\hline & WMW & $\mathrm{z}=1.33$ & $\mathrm{z}=1.36$ & $\mathrm{Z}=1.51$ & $\mathrm{z}=1.44$ & $\mathrm{z}=2.01$ \\
\hline & WN & $\mathrm{p}=0.18$ & $\mathrm{p}=0.17$ & $\mathrm{p}=0.13$ & $\mathrm{p}=0.15$ & $\mathrm{p}=0.045$ \\
\hline \multirow{4}{*}{2} & AVG (risk) & 1.53 & -1.5 & 1.524 & 0.586981 & -0.001 \\
\hline & AVG (amb) & -1.228 & 0.74 & -2.784 & -7.534 & -0.016 \\
\hline & WMW & $\mathrm{z}=1.56$ & $\mathrm{z}=-1.13$ & $\mathrm{z}=2.00$ & $\mathrm{z}=2.57$ & $\mathrm{z}=2.37$ \\
\hline & W IN W & $\mathrm{p}=0.12$ & $\mathrm{p}=0.26$ & $\mathrm{p}=0.045$ & $\mathrm{p}=0.01$ & $\mathrm{p}=0.18$ \\
\hline \multirow{4}{*}{3} & AVG (risk) & -0.62 & 1.68 & 2.478 & 0.326731 & -0.001 \\
\hline & AVG (amb) & -1.528 & -1.64127 & -1.024 & -2.84627 & -0.011 \\
\hline & WMW & $\mathrm{z}=0.85$ & $\mathrm{z}=2.27$ & $\mathrm{z}=2.12$ & $\mathrm{Z}=1.82$ & $\mathrm{z}=1.63$ \\
\hline & W N W & $\mathrm{p}=0.4$ & $\mathrm{p}=0.02$ & $\mathrm{p}=0.03$ & $\mathrm{p}=0.07$ & $\mathrm{p}=0.1$ \\
\hline \multirow{4}{*}{4} & AVG (risk) & -0.37 & 2.475 & 0.805269 & -0.43507 & -0.002 \\
\hline & AVG (amb) & 1.48 & -1.08 & -2.242 & -3.78073 & -0.069 \\
\hline & WMW & $\mathrm{z}=-1.18$ & $\mathrm{z}=1.89$ & $\mathrm{z}=1.74$ & $\mathrm{z}=1.36$ & $\mathrm{z}=1.72$ \\
\hline & & $\mathrm{p}=0.24$ & $\mathrm{p}=0.06$ & $\mathrm{p}=0.08$ & $\mathrm{p}=0.17$ & $\mathrm{p}=0.09$ \\
\hline \multirow{4}{*}{5} & AVG (risk) & 2.83 & -0.056 & -1.74646 & -0.593 & -0.002 \\
\hline & AVG (amb) & 2.336 & -0.844 & -1.49702 & -2.65327 & -0.009 \\
\hline & WMW & $\mathrm{z}=0.53$ & $\mathrm{z}=0.53$ & $\mathrm{z}=0.57$ & $\mathrm{z}=0.3$ & $\mathrm{z}=1.91$ \\
\hline & WN & $\mathrm{p}=0.59$ & $\mathrm{p}=0.6$ & $\mathrm{p}=0.57$ & $\mathrm{p}=0.76$ & $\mathrm{p}=0.06$ \\
\hline
\end{tabular}

$\mathrm{AVG}($ risk) and $\mathrm{AVG}(\mathrm{amb})$ indicate average deviations from conditional optimum (expressed in tokens). WMW: Wilcoxon-Mann-Whitney test 
Table 3: Comparing Risk and Ambiguity - Pooled Data

\begin{tabular}{lrrrrr}
\hline & \multicolumn{5}{c}{ Periods } \\
\hline & 1 & 2 & 3 & 4 & 5 \\
AVG (risk) & 0.79 & 0.43 & -0.17 & -1.83 & -0.001 \\
AVG (amb) & -0.376 & -1.53 & -3.29 & -5.66 & -0.021 \\
& & & & & \\
WMW & $\mathrm{z}=1.39$ & $\mathrm{z}=2.68$ & $\mathrm{z}=3.42$ & $\mathrm{z}=3.22$ & $\mathrm{z}=4.35$ \\
& $\mathrm{p}=0.16$ & $\mathrm{p}=0.007$ & $\mathrm{p}=0.0006$ & $\mathrm{p}=0.001$ & $\mathrm{p}=0.000$ \\
& & & & & \\
t-test & $\mathrm{t}=1.524$ & $\mathrm{t}=1.957$ & $\mathrm{t}=2.636$ & $\mathrm{t}=2.026$ & $\mathrm{t}=1.651$ \\
& $\mathrm{p}=0.065$ & $\mathrm{p}=0.026$ & $\mathrm{p}=0.005$ & $\mathrm{p}=0.023$ & $\mathrm{p}=0.051$ \\
\hline
\end{tabular}

AVG(risk) and AVG(amb) indicate average deviations from conditional optimum (expressed in tokens). WMW: Wilcoxon-Mann-Whitney test 


\section{Figures}

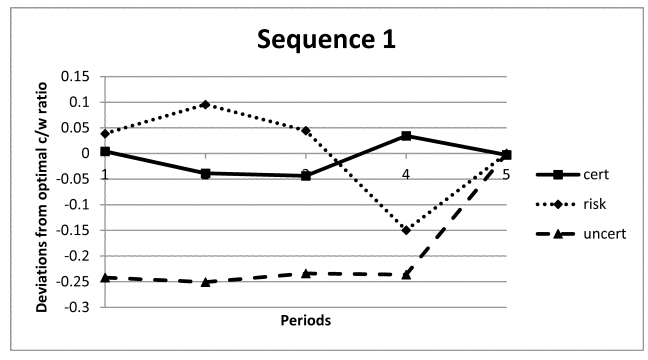

(a)

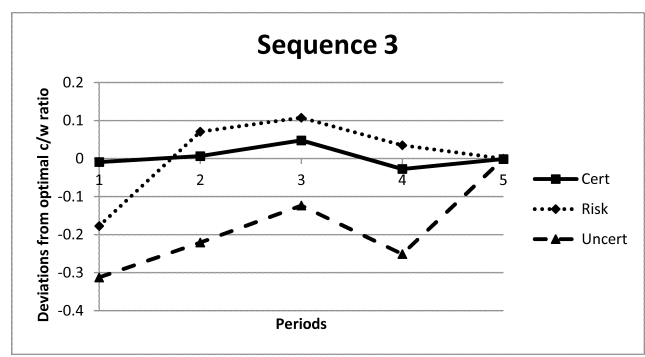

(c)

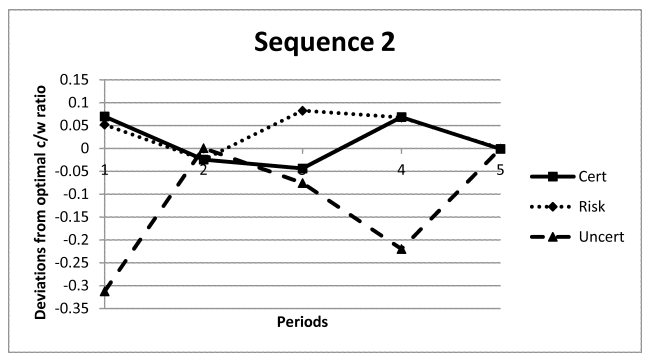

(b)

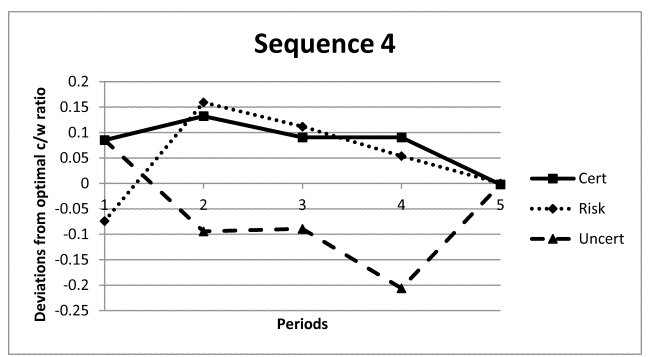

(d)

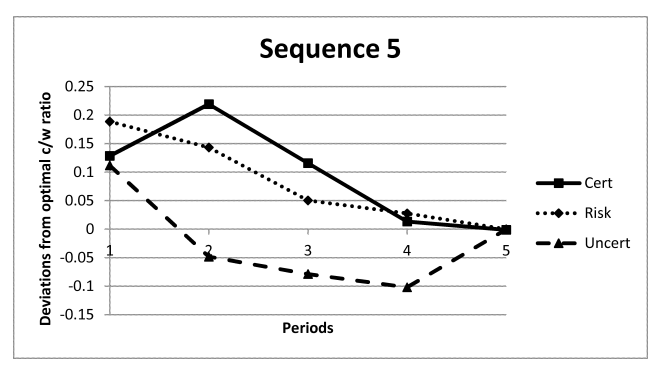

(e)

Figure 1: Deviations from optimal "c-to-w" ratio 